\title{
Approximation of the Integrals of the Gaussian Distribution of Asperity Heights in the Greenwood-Tripp Contact Model of Two Rough Surfaces Revisited
}

\author{
Radosław Jedynak ${ }^{1}$ and Jacek Gilewicz ${ }^{2}$ \\ ${ }^{1}$ Kazimierz Pulaski University of Technology and Humanities, UL. Malczewskiego 20a, 26-600 Radom, Poland \\ ${ }^{2}$ Centre de Physique Théorique, CNRS, Luminy Case 907, Aix-Marseille Université, UMR 6207, \\ Université Sud Toulon-Var, 13288 Marseille Cedex 09, France \\ Correspondence should be addressed to Radosław Jedynak; jedynakr@pr.radom.pl
}

Received 6 February 2013; Revised 6 May 2013; Accepted 7 May 2013

Academic Editor: Renat Zhdanov

Copyright (C) 2013 R. Jedynak and J. Gilewicz. This is an open access article distributed under the Creative Commons Attribution License, which permits unrestricted use, distribution, and reproduction in any medium, provided the original work is properly cited.

Some examples of application of Padé approximant techniques to approximate the integrals in question and comparison with previous results, essentially the recent Panayi-Schock and Green-wood results, show the efficiency of this simple natural approximation.

\section{Introduction}

Modelling of the contact between moving rough surfaces allows a better understanding of friction and wear mechanisms, which can be used in engineering solutions. This issue has been examined using a number of approaches. The statistical type of a contact model is still the most popular model used in rough surfaces contact. This statement does not mean that it is the best solution of contact rough surfaces. Instead of using the complete roughness data, only probability density function is used. This function means the probability of the asperity with the height between $h$ and $h+d h$. The first well-known statistical model was introduced by Greenwood and Williamson [1] (GW). They joined a statistical process with a classical Hertzian contact to deal with the rough surfaces contact. They adopted the following assumptions: (1) the asperity height distribution is Gaussian, (2) asperity contact is modelled by the Hertzian spherical contact theory, (3) the asperity tip radius is assumed constant, and (4) adhesion contact between asperities is ignored. A rough surface was described by three parameters: (1) standard deviation of asperity height distribution, (2) average asperity summit radius of curvature, and (3) areal asperity density. This model has been widely accepted and developed by numerous researchers. The main reason of its popularity is its simplicity and it's predictions are in accordance with the carried out experiments. Some interesting review articles in the frame of dry friction are written by Bhushan $[2,3]$, Buczkowski and Kleiber [4], and Jedynak and Sulek [5].

The model proposed by Greenwood and Tripp [6] (GT) extended the GW model to contact between two rough surfaces. They showed that the contact between two rough surfaces can be modelled by a contact between an equivalent single rough surface and a flat one. The equivalent rough surface is characterized by an asperity curvature and the peak-height distribution of the equivalent surface. They used the simple formula for a standard deviation of the statistical distribution as the square root of the sum of squares of the standard deviations of asperity height distributions on the two surfaces. The GT model gained large popularity in the field of elastohydrodynamic analysis, and all the following papers refer to [6]. The most frequently cited equations given by the GT model are for the following asperity contact area:

$$
A_{e}=\pi^{2}(\xi \kappa \sigma)^{2} A F_{2}(\lambda)
$$

and load carried by the following asperities:

$$
W_{e}=\frac{8 \sqrt{2}}{15} \pi(\xi \kappa \sigma)^{2} \sqrt{\frac{\sigma}{\kappa}} E^{*} A F_{5 / 2}(\lambda),
$$


where $\xi \kappa \sigma$-roughness parameter, $A$-nominal contact area,-Stribeck oil film parameter, first defined by Stribeck $[3,6]$ as $\lambda=h / \sigma, E^{*}$-effective elastic modulus, and $F_{2}, F_{5 / 2}(\lambda)$-statistical functions introduced to match the assumed Gaussian distribution of asperities.

A parameter, $\lambda$ (ratio of film thickness to the composite roughness of the contiguous surfaces), is used to ascertain any boundary contributions, which occur because of asperity interactions. It is a very important parameter for EHD analysis because interruptions in a coherent film may occur when $\lambda<3$ [7].

At this point, it is worth noting that GT equations are only valid for surfaces which have a Gaussian distribution of asperities. A lot of real engineering surfaces do not meet these requirements. For example, a typical analysis of the compression ring of a piston against the cylinder bore is not correct if we use a Gaussian distribution. Spencer et al. [8] showed that the bore usually has a liner insert, which is crosshatch honed, making a plateau geometry. This geometry provides a smooth surface to allow for hydrodynamic film buildup between the piston rings and the cylinder liner surface. On the basis of these facts we can definitely say that the mentioned surfaces do not have a Gaussian distribution of asperities ringcylinder.

On the other hand, cam-tappet contact is a good example for using the mentioned distribution. Both surfaces are well known to follow Gaussian topography. This kind of conjunction is the most loaded contact in the valve train mechanism. Jedynak and Sulek [5] showed that some kinds of galvanic coatings have also a Gaussian distribution of asperities.

Many surfaces do not follow at least a symmetric Gaussian-type distribution. Some researchers assume that the rubber-type material has a symmetric Gaussian-type distribution, but it is against the actually presented results, for example, Prokopovich et al. [9]. This paper clearly shows skew-symmetric Gaussian-type distribution for rubber seals. The mechanism of friction generation in the seal conjunction is complex, arising from adhesion of rubber in contact with the moving interface, viscous action of a thin film of fluid, and deformation of seal asperities. The authors developed a friction model based on the said mechanisms and then performed tests at both nano- and component level scales. Because the topography of the examined surfaces was nonsymmetric Gaussian, they used an alternative approach for adhesive friction, which is described in $[3,10]$. They found that adhesion is a dominant mechanism of friction.

The GT model is still being superseded by better models. Recently, Chong et al. [11] presented a friction model at fractal level, combining the effects of asperity adhesion, elastoplastic deformation, and a thin adsorbed boundary active molecular layer. This model forms the basis for the development of a generic multispecies lubricant-random roughness surface methodology. They stated that traditional boundary friction models do not apply to randomly distributed topology.

Statistical functions $F_{n}(h)$ which are used in extended GT and GW models have the following form:

$$
F_{n}(h)=\frac{1}{\sqrt{2 \pi}} \int_{h}^{\infty}(s-h)^{n} e^{-s^{2} / 2} d s, \quad n=\frac{1}{2}, 1, \frac{3}{2}, 2, \frac{5}{2} .
$$

TABLE 1: Computed values of $F_{5 / 2}(h)$ used to build the approximation formulas.

\begin{tabular}{lcc}
\hline$h$ & Old values [6] & Improved values [14] \\
\hline 0 & .61664 & .616634 \\
.5 & .24040 & .240402 \\
1 & .08056 & .0805623 \\
1.5 & .02286 & .0228631 \\
2 & .00542 & .00542371 \\
2.5 & .00106 & .00106369 \\
3 & .00017 & .0001709 \\
3.5 & .00002 & .00002231 \\
4 & .00000 & .000002354 \\
4.5 & - & .0000002 \\
5 & - & .0000000 \\
$\vdots$ & $\vdots$ & $\vdots$ \\
6.5 & & $10^{-12}$ \\
\hline
\end{tabular}

This function can only be solved using numerical methods and that is why we discuss the most efficient approximations formulas for $F_{5 / 2}(h)$ in the next chapter. They are used by many researchers in elastohydrodynamic analysis, In this case $F_{5 / 2}$ is a function of $\lambda$. Then we propose a new formula which is based on Padé approximation method.

It is important to emphasize that the approximations discussed next can be only applicable to surfaces which have a Gaussian distribution of asperities but not for all engineering surfaces which actually exist.

\section{Previous Approximations}

The GT model required the numerical integration of the Gaussian distribution. Greenwood and Tripp [6] provided tabulated results for this integral over its effective range: 9 points on the interval $[0,4], 0 \leq F_{n}<1$ for all $n$, the last digits given in [6] corresponded to $10^{-5}$ and from $h=4$ these values were as follows:

$$
\begin{gathered}
F_{3 / 2}(4)=F_{2}(4)=F_{5 / 2}(4)=0.00000, \\
F_{n}(h>4)=0 .
\end{gathered}
$$

These values were recently used by a number of authors $[7$, 12,13 ] to approximate the integrals $F_{n}$ by simpler functions. Unfortunately, all these authors used old tabulated values, which were recently corrected [14] as shown in Table 1 for $F_{5 / 2}(h)$.

The following models of approximation of $F_{5 / 2}$ based on the coefficient fitting to the tabulated values were proposed:

(i) Hu et al. power law approximation [13]:

$$
\begin{gathered}
F_{5 / 2}^{(C)}(h)=\alpha(4.0-h)^{\beta} \quad h<4.0, \\
F_{5 / 2}(h \geq 4)=0,
\end{gathered}
$$




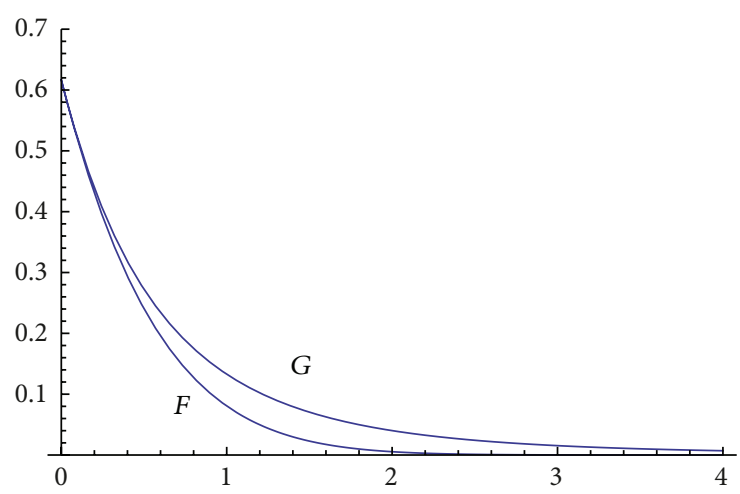

FIGURE 1: Graphs of $F_{5 / 2}(h)$ and $G_{5 / 2}(h)$ for $h \in[0,4]$.

(ii) Teodorescu et al. 5-degree polynomial approximation [7]:

$$
\begin{gathered}
F_{5 / 2}^{(A)}(h)=a_{0}+a_{1} h+\cdots+a_{5} h^{5} \quad h<4.0, \\
F_{5 / 2}(h \geq 4)=0,
\end{gathered}
$$

(iii) Panayi and Schock exponential form [12]:

$$
\begin{gathered}
F_{5 / 2}^{(P)}(h)=e^{\left(-.48347-1.65118 h+.06043 h^{2}\right) /\left(1-.19256 h+.01610 h^{2}\right)} \\
-.00000350796 \text { for } h<4.0 \\
F_{5 / 2}^{(P)}(h \geq 4)=0
\end{gathered}
$$

(iv) Greenwood quartic fit to $\ln F_{n}[14]$ :

$$
\begin{aligned}
F_{5 / 2}^{(G)}(h)= & .616634 \\
& \times e^{.00121735 h^{4}-.02342695 h^{3}-.2696729 h^{2}-1.7433954 h} .
\end{aligned}
$$

The paper [14] contains the analysis by Greenwood of the paper [12], the corrected tabulated values of $F_{5 / 2}$, and the model (7), which remains better than the Panayi-Schock model even after the correction of the coefficients in (6) by the new values of $F_{5 / 2}$. Greenwood proposed also in [14] to consider the following modified function:

$$
G_{n}(h)=e^{h^{2} / 2} F_{n}(h)
$$

which is more adapted to the numerical treatment, because it is slower decreasing than $F_{n}$, as shown in Figure 1 in the case of $n=5 / 2$. Figure 1 shows both function $F_{5 / 2}(h)$ and $G_{5 / 2}(h)$ for $h \in[0,4]$.

\section{Padé Approximation (PA) of $F_{5 / 2}$ or of $G_{5 / 2}$}

The authors of the present paper are surprised that no Padé approximation was tried to approximate $F_{5 / 2}$ or $G_{5 / 2}$ or to approximate the integrated function. It is an automatic and low-cost method leading frequently to the surprisingly good results.
Let $f$ be an analytic function at $N$ real different points having the following power expansions:

$$
\sum_{k=0}^{\infty} c_{k}\left(x_{j}\right)\left(x-x_{j}\right)^{k}, \quad j=1, \ldots, N .
$$

In practical situations, we know only a few first coefficients of each expansion, and then we have to deal with the limited information characterized by the following truncated power series:

$$
\sum_{k=0}^{p_{j}-1} c_{k}\left(x_{j}\right)\left(x-x_{j}\right)^{k}+O\left(\left(x-x_{j}\right)_{j}^{p}\right), \quad j=1, \ldots, N .
$$

The $N$-point Padé approximant (NPA) to $f$, if it exists, is a rational function $P_{m} / Q_{n}=[\mathrm{m} / \mathrm{n}]$ denoted, if it is needed, as follows:

$$
\begin{gathered}
{\left[\frac{m}{n}\right]_{x_{1} x_{2} \cdots x_{N}}^{p_{1} p_{2} \cdots p_{N}}(x)=\frac{a_{0}+a_{1} x+\cdots+a_{m} x^{m}}{1+b_{1} x+\cdots+b_{n} x^{n}}} \\
m+n+1=p=p_{1}+p_{2}+\cdots+p_{N}
\end{gathered}
$$

satisfying the following relations:

$$
f(x)-\left[\frac{m}{n}\right](x)=O\left(\left(x-x_{j}\right)^{p_{j}}\right), \quad j=1,2, \ldots N .
$$

Each $p_{j}$ represents the number of coefficients $c_{k}\left(x_{j}\right)$ of expansion actually used for the computation of NPA. In the present problem, all $p_{j}=1$, and the construction of NPA $[m / n]$ is equivalent to the construction of the rational interpolation $P_{m} / Q_{n}$. The classical Padé approximant (PA) is a one-point PA computed from the development of the function $f$ at the origin $x=0$. It is defined by the linear system obtained by the definition (13) with right-hand side $O\left(x^{m+n+1}\right)$.

At the first time, let us estimate crudely the function $F_{5 / 2}$ by an exponential function in the interval $[0,4]$ :

$$
\begin{gathered}
F_{5 / 2}(h)=\alpha e^{-\beta h ;} \\
\alpha=.616634 ; \\
\beta=-\frac{1}{4} \ln \frac{.000002354}{.616634} .
\end{gathered}
$$

Of course this simple interpolation over two nodes badly reproduces $F_{5 / 2}$ on this interval; however, it shows that our Padé approximant must decrease on $[0,4](8)$ and must become rapidly zero after. That is, the degree of its denominator must be sensibly greater than those of numerator.

To obtain the optimal results using the Padé approximation method, one must select the "best" PA $[\mathrm{m} / \mathrm{n}]$; it means the "best" degrees $m$ and $n$. Many practical algorithms of choice of the best PA were proposed in [15]. For the present problem, we select two following algorithms: $\rho$-method and the method of valleys in $c$-table, both based on the analysis of the coefficients of the expansion of the considered function $f(x)=\sum c_{j} x^{j}$. The $\rho$-method analyze the sequence 
TABle 2

\begin{tabular}{ccccccccc}
\hline$m$ & 0 & 1 & 2 & 3 & 4 & 5 & 6 & 7 \\
\hline 0 & 1 & .61 & .38 & .23 & .145 & $.89-1$ & $.55-1$ & $.34-1$ \\
1 & 1 & -1.08 & .49 & -.12 & $.15-1$ & $-.47-3$ & $-.72-4$ & $.104-5$ \\
2 & 1 & 1.08 & .30 & $.29-1$ & $.11-2$ & $.14-4$ & $.10-6$ & $.2-8$ \\
3 & 1 & -.81 & .12 & $-.45-2$ & $.53-4$ & $-.19-6$ & $.16-9$ & \\
4 & 1 & .49 & $.33-1$ & $.48-3$ & $.18-5$ & $.2-8$ & & \\
5 & 1 & -.26 & $.75-2$ & $-.38-4$ & $.42-7$ & & & \\
6 & 1 & .12 & $.14-2$ & $.24-5$ & & & & \\
7 & 1 & -.053 & $.22-3$ & & & & & \\
8 & 1 & .021 & & & & & & \\
\hline
\end{tabular}

$\left\{\rho_{n}=\left|c_{n} / c_{n+1}\right|\right\}$. The nonmonotonicity of the few first terms of $\left\{\rho_{n}\right\}$, if the presumed PA is such that $m>n$, indicates the difference $m-n$. The $c$-table is the table of determinants of matrices of linear systems defining the denominators of the classical (one-point) PA $[m / n]$ :

$$
C_{n}^{m}=\left|\begin{array}{cccc}
c_{m} & c_{m-1} & \cdots & c_{m-n+1} \\
c_{m+1} & c_{m} & \cdots & \cdots \\
\vdots & \vdots & \vdots & \vdots \\
c_{m+n-1} & \cdots & \cdots & c_{m}
\end{array}\right|
$$

At the same time, the dominant error of $\{f-[m / n]\}$ near the origin is proportional to $C_{n}^{m}$. The minima of each antidiagonal in the table of $\left|C_{n}^{m}\right|$, of the entries builded with the same information, indicate the paradiagonal with fixed $m-n$ of the best PA.

By the integration of parts of (3), one obtains the following recurrence formulae:

$$
F_{n+1}(h)=n F_{n-1}(h)-h F_{n}(h) .
$$

The following formulas for the derivatives are also useful to obtain the development in power series of $F_{n}$ :

$$
F_{n}^{\prime}(h)=-h F_{n}(h)-F_{n+1}(h)=-n F_{n-1}(h) .
$$

Now we can compute the expansion in the power series of $F_{5 / 2}$ near the origin, where $a:=F_{1 / 2}(0)=.411089$ and $b:=$ $F_{3 / 2}(0)=.43002$ :

$$
\begin{aligned}
F_{5 / 2}(h)= & \left(\frac{3 a}{2}\right)-\left(\frac{5 b}{2}\right) h+\left(\frac{15 a}{8}\right) h^{2}-\left(\frac{5 b}{8}\right) h^{3} \\
& +\left(\frac{5 a}{64}\right) h^{4}+\left(\frac{b}{64}\right) h^{5}-\left(\frac{a}{256}\right) h^{6} \\
& -\left(\frac{5 b}{5376}\right) h^{7}+\cdots \\
= & .616634-1.07505 h+.770793 h^{2}-.268762 h^{3} \\
& +.0321164 h^{4}+.00671906 h^{5} \\
& -.00160582 h^{6}-.000399944 h^{7} \\
& +.000100364 h^{8}+.0000249965 h^{9}+\cdots,
\end{aligned}
$$

and the power series expansion of the function $G_{5 / 2}$ :

$$
\begin{aligned}
G_{5 / 2}(h)= & e^{h^{2} / 2} F_{5 / 2}(h)=.61663422 \\
& -1.07505 h+1.07911 h^{2}-.8062875 h^{3} \\
& +.49459203 h^{4}-.262043 h^{5}+.12364801 h^{6} \\
& -.0530326 h^{7}+.0209760 h^{8}-.0077339 h^{9}+\cdots .
\end{aligned}
$$

The $\rho$-method consists of the analysis of the regularity of the sequence $\left\{\rho_{n}=\left|c_{n} / c_{n+1}\right|\right\}$. The beginning of the sequence $\left\{\rho_{n}\right\}$ corresponding to (19) is regular:

$$
\rho_{0}, \rho_{1} \ldots \rho_{7}=.57, .996,1.34,1.63,1.89,2.12,2.33,2.53 \text {. }
$$

This indicates that the rational approximation $\{m / n\}$ corresponding to this series is such that $m \leq n$. More significant result is observed studying the $c$-table corresponding to (19), where the notation $.1-5$ means $0.1 \times 10^{-5}$ (see Table 2).

The minima are located on the positions $0 / 3,1 / 4,2 / 5$ and $3 / 6$. The best PA is then as follows:

$$
\begin{aligned}
{\left[\frac{3}{6}\right]_{G}(h)=(} & .616634+.0587436 h \\
& \left.+.0186433 h^{2}-.0000525721 h^{3}\right) \\
\times & \left(1 .+1.83868 h+1.48582 h^{2}+.680187 h^{3}\right. \\
& \left.+.187771 h^{4}+.0300186 h^{5}+.0022127 h^{6}\right)^{-1} .
\end{aligned}
$$

In Table 3 we compare the exact values of $F_{5 / 2}(h)$ with those obtained by multiplying $[3 / 5]_{G}$ and $[3 / 6]_{G}$ by $e^{-\left(h^{2} / 2\right)}$ and with the values calculated by the Greenwood approximation [14] and the Panayi approximation [12].

Clearly the [3/6] PA leads to the best values, but it is computed with 10 coefficients (information) on $G_{5 / 2}$ at $h=0$. The results obtained with [3/5] PA (9 information) are better in the range $0 \div 2.5$ than the Greenwood results (obtained also with 9 information). It is natural, because the PA calculated 
TABle 3: Approximation of $F_{5 / 2}$ via one point $(h=0)$ Padé approximants of $G_{5 / 2}$.

\begin{tabular}{|c|c|c|c|c|c|}
\hline$h$ & $\operatorname{exact} F_{5 / 2}(h)$ & $e^{-h^{2} / 2}[3 / 5]_{G}(h)$ & $e^{-h^{2} / 2}[3 / 6]_{G}(h)$ & Greenwood & Panayi \\
\hline 0. & .616634 & .616634 & .616634 & .616634 & .616636 \\
\hline .5 & .240402 & .240402 & .240402 & .240401 & .240395 \\
\hline 1. & .0805623 & .0805623 & .0805623 & .0805597 & .0805637 \\
\hline 1.5 & .0228631 & .0228627 & .022863 & .0228629 & .0228449 \\
\hline 2. & .00542371 & .00542304 & .00542366 & .00542412 & .00541762 \\
\hline 2.5 & .00106369 & .00106315 & .00106365 & .00106379 & .00106965 \\
\hline 3. & .000170873 & .000170616 & .000170852 & .000170864 & .000175778 \\
\hline 3.5 & .0000223124 & .0000222326 & .0000223059 & .000022307 & .0000225912 \\
\hline 4. & $2.35338-6$ & 2.33623-6 & 2.35197-6 & $2.35373-6$ & $1.17725-9$ \\
\hline
\end{tabular}

The bold digits are exact.

TABLE 4: 9-point Padé approximation of $F_{5 / 2}$ obtained directly and also via approximation of $G_{5 / 2}$. Comparison with Greenwood and Panayi results.

\begin{tabular}{|c|c|c|c|c|c|}
\hline$h$ & exact $F_{5 / 2}(h)$ & {$[3 / 5]_{F}(h)$} & $e^{-h^{2} / 2}[3 / 5]_{G}(h)$ & Greenwood & Panayi \\
\hline 0. & .616634 & .616634 & .616634 & .616634 & .616636 \\
\hline .25 & .391978 & .3849 & .391977 & .39198 & .391941 \\
\hline .5 & .240402 & .240402 & .240402 & .240401 & .240395 \\
\hline .75 & .141962 & .142463 & .141962 & .141959 & .141971 \\
\hline 1. & .0805623 & .0805623 & .0805623 & .0805597 & .0805637 \\
\hline 1.25 & .0438561 & .0438004 & .0438561 & .0438548 & .0438441 \\
\hline 1.5 & .0228631 & .0228631 & .0228631 & .0228629 & .0228449 \\
\hline 1.75 & .0113963 & .0114051 & .0113963 & .0113967 & .0113818 \\
\hline 2. & .00542371 & .00542371 & .00542371 & .00542412 & .00541762 \\
\hline 2.25 & .00246124 & .00245933 & .00246124 & .0024615 & .00246296 \\
\hline 2.5 & .00106369 & .00106369 & .00106369 & .00106379 & .00106965 \\
\hline 2.75 & .00043732 & .000437939 & .00043732 & .000437338 & .000443886 \\
\hline 3. & .000170873 & .000170873 & .000170873 & .000170864 & .000175778 \\
\hline 3.25 & .0000633931 & .0000630551 & .0000633931 & .0000633825 & .0000658778 \\
\hline 3.5 & .0000223124 & .0000223124 & .0000223124 & .000022307 & .0000225912 \\
\hline 3.75 & $7.44495-6$ & $.7 .44495-6$ & 7.44495-6 & $7.44359-6$ & $6.11165-6$ \\
\hline \multirow[t]{3}{*}{4.} & $2.35338-6$ & $2.35338-6$ & $2.35338-6$ & $2.35373-6$ & $1.17725-9$ \\
\hline & & & standard dev.: & stand. dev.: & stand. dev.: \\
\hline & & & $9.6-18$ & $9.3-7$ & $7.3-6$ \\
\hline
\end{tabular}

The bold digits are exact.

from the development of a function near the origin gives good results in the neighborhood of the origin. In the following, we will compare the $N$-point PA $[3 / 5]_{h_{1} h_{2} \cdots h_{9}}^{11 \cdots 1}$ and $[3 / 6]_{h_{1} h_{2} \cdots h_{9}}^{21 \cdots 1}$ with other results. The NPA [3/6], corresponding to our best choice, is easily calculated with 9 values of $G_{5 / 2}$ and one additional coefficient of development (19):

$$
\begin{aligned}
& {\left[\frac{3}{5}\right]_{G}(h)=(.616634+.10073 h} \\
&\left.+.00818188 h^{2}-.000161362 h^{3}\right)
\end{aligned}
$$

$$
\begin{gathered}
\times\left(1 .+1.90693 h+1.58667 h^{2}+.740254 h^{3}\right. \\
\left.+.19751 h^{4}+.0298511 h^{5}\right)^{-1} .
\end{gathered}
$$

Table 4 contains the values of the approximations also in the points located between the nodes, because the NPA are, of course, exact in the nodes, and we must analyze other points to compare our approximations with those of Greenwood and Panayi. The advantage to determine the values of $F_{5 / 2}(h)$ via $e^{-h^{2} / 2}[3 / 5]_{G}(h)$ appears evident. In fact, the last values have in 


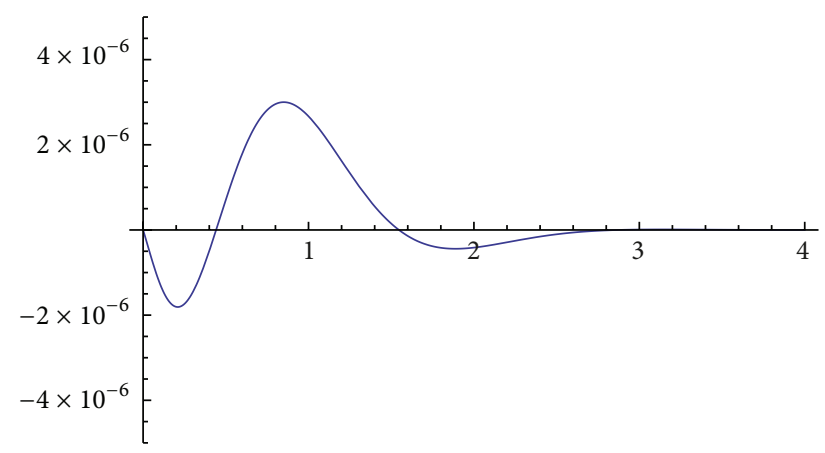

Figure 2: Graph of the error of Greenwood (7) approximation: $F_{5 / 2}(h)-F_{5 / 2}^{(G)}(h)$.

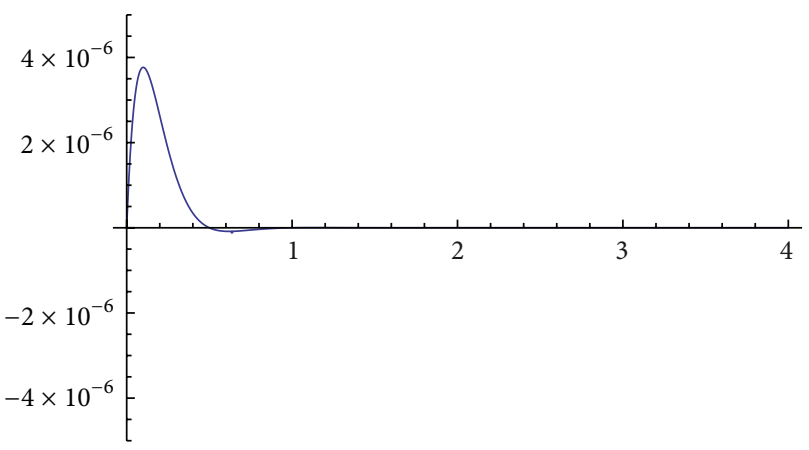

FIGURE 3: Graph of the error of our approximation: $F_{5 / 2}(h)-$ $e^{-\left(h^{2} / 2\right)}[3 / 5]_{G}(h)$.

the intermediate points all digits exact and it is not the case of the values of the NPA $[3 / 5]_{F}(h)$ determined directly by the values given in [14] (Table 1):

$$
\begin{aligned}
{\left[\frac{3}{5}\right]_{F}(h)=(} & .616634-.457287 h \\
& \left.+.1141502 h^{2}-.0095798 h^{3}\right) \\
\times & \left(1 .+1.3788066 h-.997978 h^{2}+2.988216 h^{3}\right. \\
& \left.-1.651924 h^{4}+.5588187 h^{5}\right)^{-1}
\end{aligned}
$$

Figures 2 and 3 illustrate this fact clearly.

Here, we see that the simple NPA [3/5], that is, an NPA builded with the same information that Greenwood or Panayi approximations, is the best. The comparison of the standard deviations is significant. Other advantage consists in its simple calculation. The following figures representing the error of our approximations illustrate the sharpness of our results.

It is possible to try other approximations using the Padé techniques. One can use other interpolation nodes for $h>$ 4 with values $F_{5 / 2}(h)=0$ and in particular the point at infinity $F_{5 / 2}(\infty)=0$. For instance, if we compute 11 points Padé approximant $[m / n](m+n+1=11)$, we can choose $[0 / 10],[1 / 9],[2 / 8],[3 / 7],[4 / 6], \ldots$ where the behavior at infinity is, respectively:

$$
\frac{\alpha_{1}}{h^{10}}, \frac{\alpha_{2}}{h^{8}}, \frac{\alpha_{3}}{h^{6}}, \frac{\alpha_{4}}{h^{4}}, \frac{\alpha_{5}}{h^{2}}, \ldots,
$$

where $\alpha_{i}$ can be crudely estimated at $h=4$ as follows:

$$
\alpha_{1}=.000002354 \times 4^{10}=2.36 ; \ldots ; \alpha_{5}=.000002354 \times 4^{2} .
$$

The numerical tests show that simple use of these additional nodes does not improve the approximation. The effect of appearing oscillations is negative.

The Padé approximant method allows, if it is needed, to obtain the two-sided estimates of the approximated function. This consists of construction of one NPA $[m / n]$ from $K=$ $m+n+1$ given coefficients and of the second one, $[m-1 / n]$ or $[m / n-1]$, with $K-1$ coefficients. In this case, if the first NPA is greater than approximated function between two nodes, the second is less than this function. Both NPA bound the approximated function from top and from below [16].

\section{Conclusion}

The efficiency of one-point as well as $N$-point Padé approximation method applied to the special tribology problem is presented and compared with other methods. This method starts from the choice of the best Padé approximant and is easily feasible. Our results confirm an ingenious idea of Greenwood proposing to approximate a modified function $G_{n}$, more smooth that the function $F_{n}$ (see Figure 1 ), and then return to the calculus of $F_{n}$, which allows to reduce considerably the numerical errors.

\section{Acknowledgment}

The authors wish to thank the reviewers for their very constructive and helpful comments which are important to improve the paper.

\section{References}

[1] J. A. Greenwood and J. B. P. Williamson, "Contact of nominally at surfaces," Proceedings of the Royal Society A, vol. 295, pp. 300319, 1966.

[2] B. Bhushan, "Contact mechanics of rough surfaces in tribology: multiple asperity contact," Tribology Letters, vol. 4, no. 1, pp. 135, 1998.

[3] B. Bhushan, Handbook of Micro/Nanotechnology, CRC Press, 1999.

[4] R. Buczkowski and M. Kleiber, "Statistical models of rough surfaces for finite element 3D-contact analysis," Archives of Computational Methods in Engineering, vol. 16, no. 4, pp. 399424, 2009.

[5] R. Jedynak and M. Sulek, "Numerical and experimental investigation of plastic interaction between rough surfaces," Arabian Journal for Science and Engineering. In press.

[6] J. A. Greenwood and J. H. Tripp, "The contact of two nominally at rough surfaces," Proceedings of the Institution of Mechanical Engineers, vol. 185, no. 1970-1971, pp. 625-634. 
[7] M. Teodorescu, M. Kushwaha, H. Rahnejat, and S. J. Rothberg, "Multi-physics analysis of valve train systems: from system level to microscale interactions," Journal of Multi-Body Dynamics, vol. 221, no. 3, pp. 349-361, 2007.

[8] A. Spencer, A. Almqvist, and R. Larsson, "A numerical model to investigate the effect of honing angle on the hydrodynamic lubrication between a combustion engine piston ring and cylinder liner," Journal of Engineering Tribology, vol. 225, no. 7, pp. 683-689, 2011.

[9] P. Prokopovich, S. Theodossiades, H. Rahnejat, and D. Hodson, "Friction in ultra-thin conjunction of valve seals of pressurised metered dose inhalers," Wear, vol. 268, no. 5-6, pp. 845-852, 2010.

[10] R. Gohar and H. Rahnejat, Fundamentals of Tribology, Imperial College Press, 2008.

[11] W. W. F. Chong, M. Teodorescu, and H. Rahnejat, "Nanoscale elastoplastic ad-hesion of wet asperities," Journal of Engineering Tribology, 2013.

[12] A. P. Panayi and H. J. Schock, "Approximation of the integral of the asperity height distribution for the Greenwood-Tripp asperity contact model," Journal of Engineering Tribology, vol. 222, no. 2, pp. 165-169, 2008.

[13] Y. Hu, H. S. Cheng, T. Arai, Y. Kobayashi, and S. Aoyama, "Numerical simulation of piston ring in mixed lubrication-a nonaxisymmetrical analysis," Journal of Tribology, vol. 116, no. 3, pp. 470-478, 1994.

[14] J. A. Greenwood, "Approximation of the integral of the asperity height distribution for the Greenwood-Tripp asperity contact model," Journal of Engineering Tribology, vol. 222, no. 7, pp. 995996, 2008.

[15] J. Gilewicz, Approximants de Padé, vol. 667 of Lecture Notes in Mathematics, Springer, Berlin, Germany, 1978.

[16] R. Jedynak and J. Gilewicz:, "Approximation of smooth functions by weighted means of N-point Pade approximants," Ukrainian Mathematical Journal. In press. 


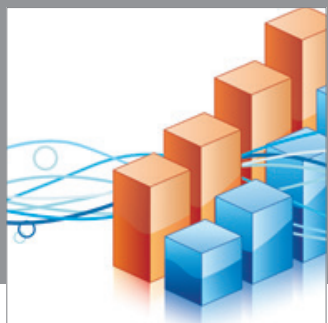

Advances in

Operations Research

mansans

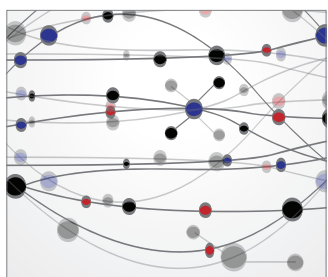

The Scientific World Journal
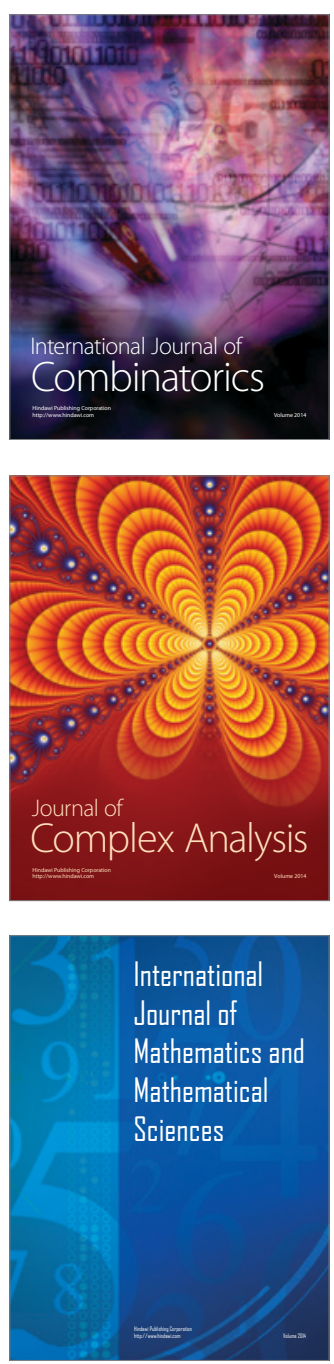
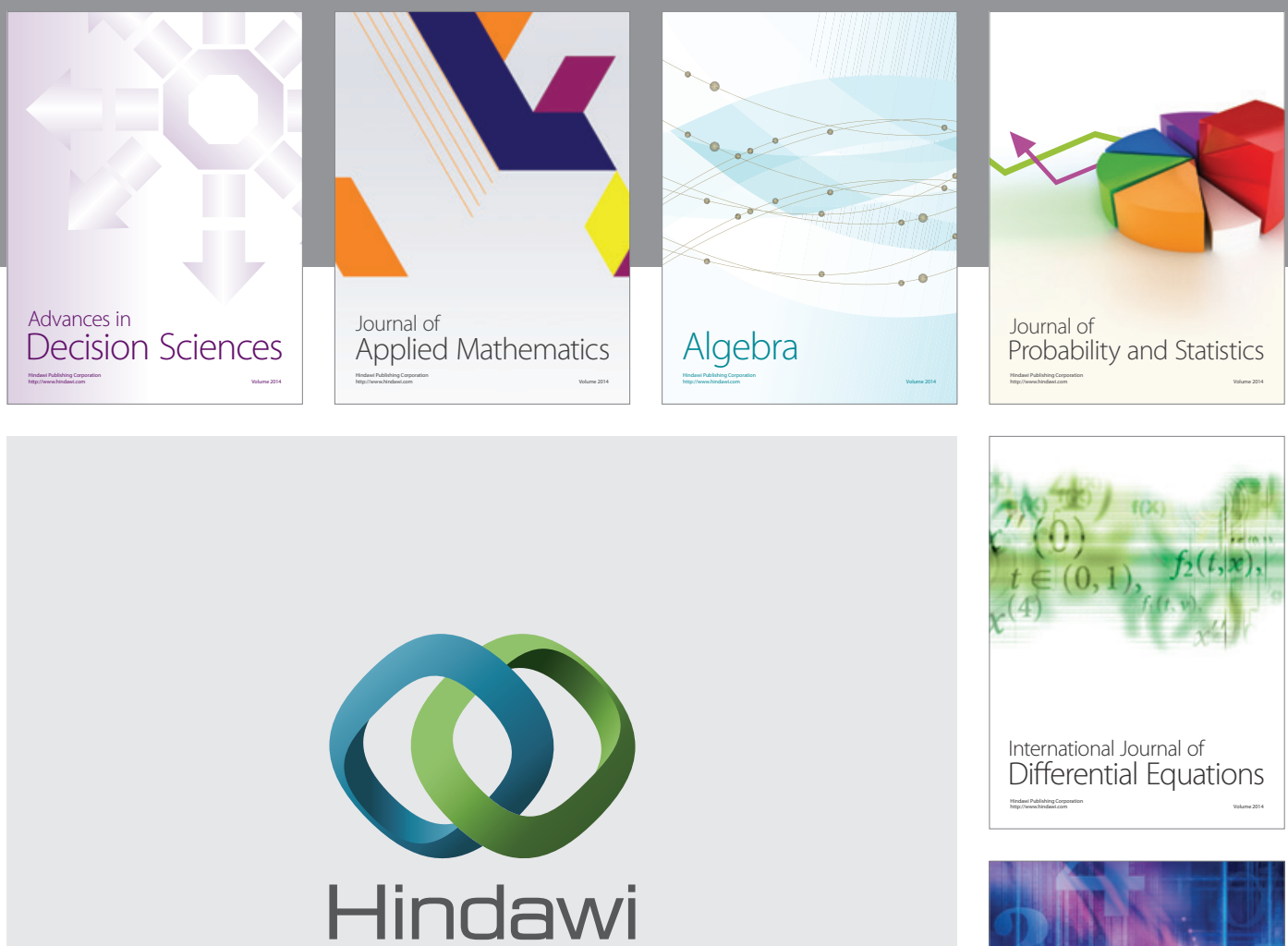

Submit your manuscripts at http://www.hindawi.com
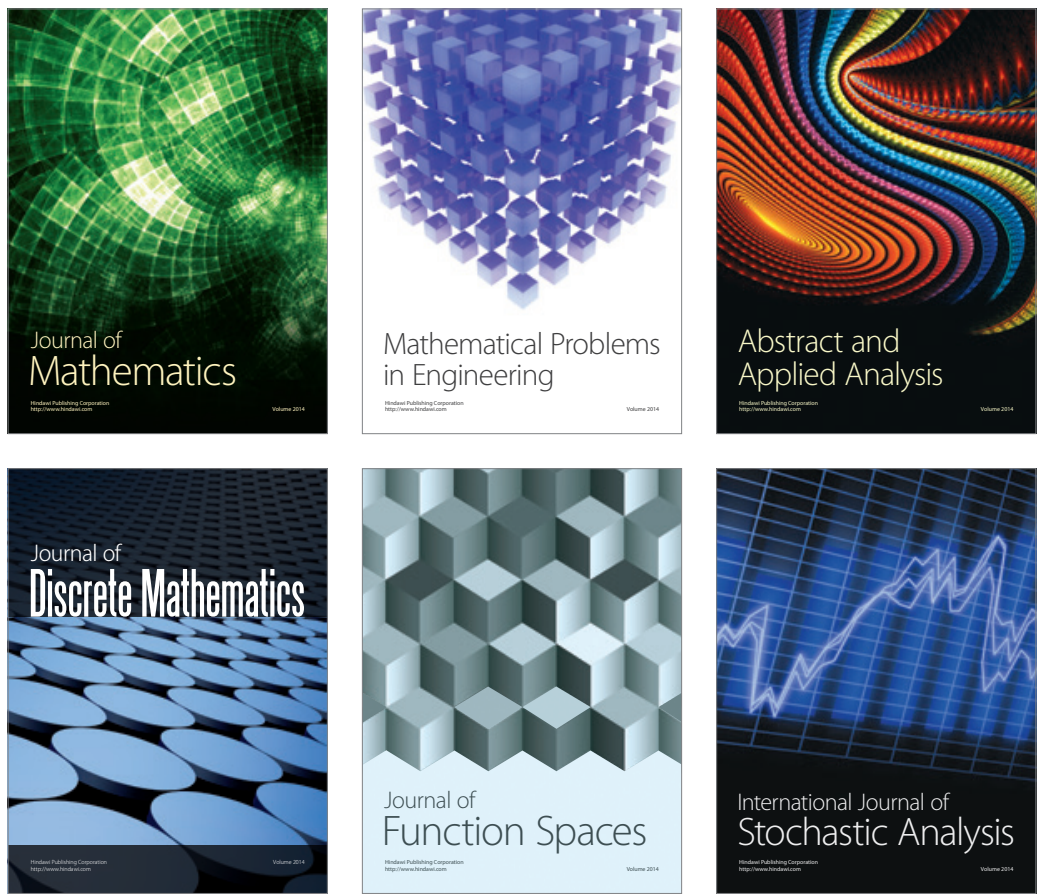

Journal of

Function Spaces

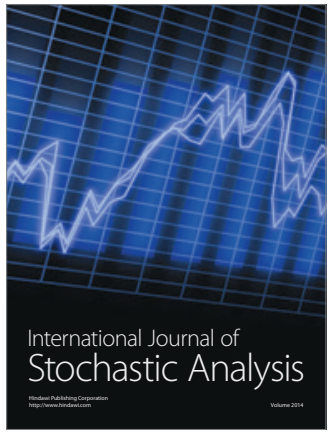

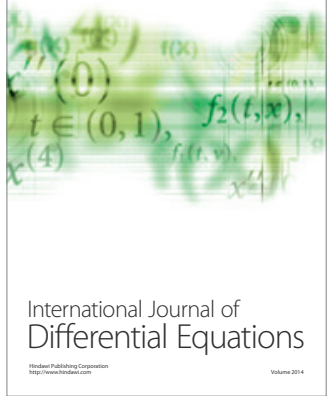
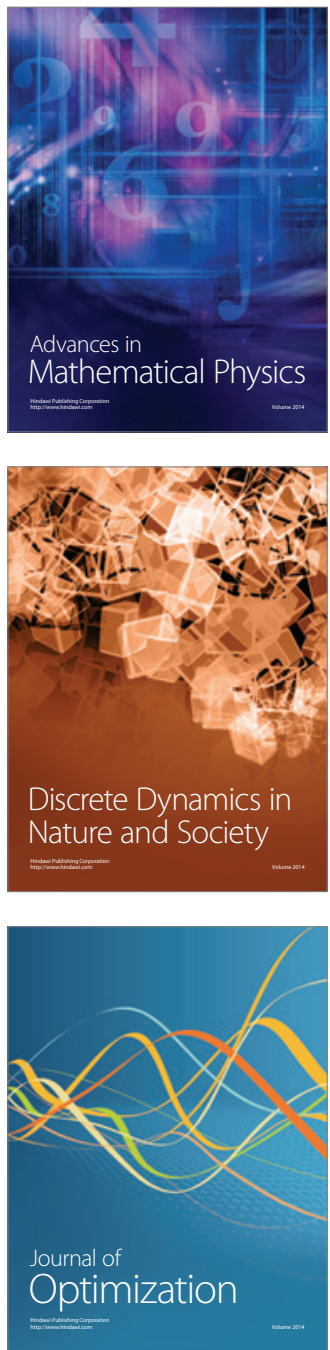